\title{
Intelligent Medical Diagnostic Systems for Arthritis
}

\author{
Sujeet More, Jimmy Singla
}

\begin{abstract}
Arthritis is a chronic, an autoimmune disease which may affect in many critical health problems in patients. From the past few years, the number of patients suffering from arthritis are swiftly escalating. Until now there is no accurate treatment discovered for this rare disease. The paper discusses the different machine learning techniques in early detection of Arthritis so that, early diagnosis can help the patients to detect/ cure the disease.
\end{abstract}

Keywords: Machine Learning, Disease diagnosis, Rheumatoid Arthritis, Deep learning.

\section{INTRODUCTION}

In medical, the disease is like an additional health problem is not a complete methodological idea. Undeniably, each and every person own there remembrance or distinctive knowledge of illness which has been long and one of the foremost human concerns [1]. Medical field defines sickness or disease as harm or infirmity of consistent functioning of the human body that is characterized by broadly sign and symptoms [2,3]. Disease is combination of visible sign and indications, that had better be construed by doctors in proper way. This course of action has been adapted by the analysis and diagnosis. Analysis of disease is standard, intricate and tough procedure in healthcare specialists, for that reason, the doctors simultaneously considers the numerous reasons and conditions with respect to medical signs [6]. Disease diagnosis in a healthcare situation can't be specified with any specific definition, but, in over-all healthcare, it discusses the complex procedure of conclusion building, which leads to a clear-cut empathetic of patient's healthiness issue [4]. Disease analysis is essential in clinical decision creation, which encompasses diverse individual and unbiassed factors. Therefore, an timely suitable and a clearly defined diagnosis has the utmost vital role in decisive disease or disorder. Henceforth, until a conclusive diagnosis is not determined, the treatment strategy cannot be determined [5].

Since obtaining truthful analysis, is the main facet of healthcare topics, many investigators practically used numerous scientific techniques in the progress of infirmity investigation for serving health centres to make the utmost precise results [9-12]. Investigators outline the medical healthcare process in diverse formats to overwhelmed the vagueness of illness analysis. Even though, it is very tough to describe all the real world proofs in terms of crisp values, a novel technique has to be proposed to model this vague. As a outcome, machine learning was defined practically to model uncertainty in medicine. Machines learning utilizes few convinced algorithms, as hidden insights can be initiated from statistics, here we don't train the machines, but repetitive learning process of ML permits the machines to acclimate its systems and results as it is given to novel circumstances and information.

Specifically, machine learning helps and provides extensive social influences in medical healthcare realm. The budding industry of electronic devices like smartwatches and other electronic devices, constantly gather/ collect a glut of health data, which proves acts as proper resolution for both tumbling the expanding amount of healthcare and serving to create a healthier patient and doctor association. The key objective of the paper, investigating different studies in machine learning with respect to arthritis (A enduring challenging illness categorized by joint swelling, joint tenderness, and destruction of synovial joints, leading to severe disability and premature mortality) diagnosis, through a systematic review.

The CDC (Centres for Disease Control and Prevention) in [7] uses collective information collected on or after with National Health Interview Survey (NHIS) years 2013-2015 Sample Adult core components to estimate average yearly arthritis occurrence in the civilian, noninstitutionalized US adult population aged 18 years or older. Commonly, the projected $22.7 \%$ (54.4 million) of adults had specialist diagnosed arthritis, with knowingly higher age adjusted occurrence in women $(23.5 \%)$ than in men $(18.1 \%)$. Arthritis occurrence increases with growing age.

The remaining review article is ordered in following way: Section 2 introduces a brief literature analysis of machine learning applied to illness/ disease identification. Section 3 introduces the proposed method and description of it. Section 4 describes an analysis of articles. Section 5 indicates the results and analysis based on defined objectives. Section 6 presents the interpretation and future plan.

Revised Version Manuscript Received on 16 September, 2019.

Sujeet More, Research scholar, School of CSE, Lovely Professional University Punjab

Jimmy Singla, Associate Professor, School of CSE, Lovely Professional University Punjab.jimmy.21733@1pu.co.in 


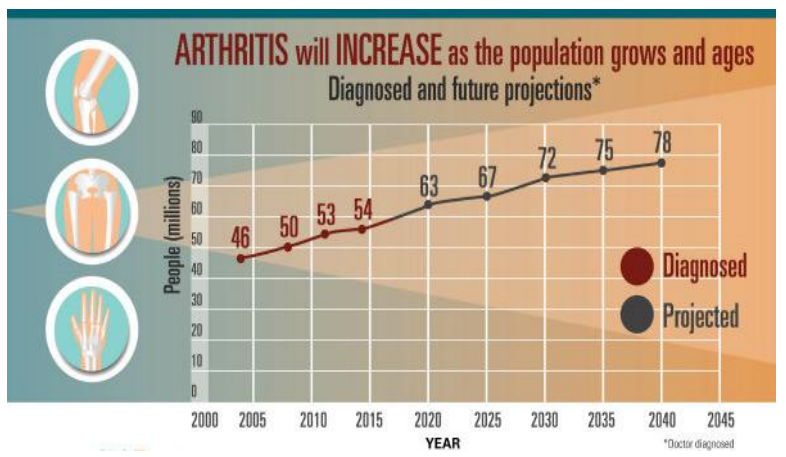

Fig 1: Arthritis patients growth from 2000 to 2045

Source: 2013 to 15 National Health Interview Survey

\section{LITERATURE REVIEW}

Healthcare, one of the reckless rising sectors nowadays, huge research has been done. Some of the research reviews are as follows:

Dr. Neeraj et al. in [14] presented a framework to classify and predict the patients with arthritis disease. The framework uses datasets collected from Koch (1998) with double blind clinical trial. The framework classifies the data records with features like id, age, sex and treatment with an algorithm CART to find true positive, false positive rates. Min Chen et al. in [15] presented a new algorithm based on CNN i.e. CNN-MDRP. The system uses hospital data (2013 to 2015) with 31919 patients, to find cerebral infraction disease with an accuracy of $94.80 \%$. The Neural Network algorithm presented by Dong et al. [16] helps to find genetic modules for arthritis disease with real dataset GSE54629. The system uses the gene to biomarks for treatment in Rheumatoid arthritis to the precise analysis of biological pathways with dynamic tree cut algorithm. The presence of Rheumatoid arthritis and its identification using thermal imaging and to automatic segment, the abnormal region in the knee, Gopikrishnan [17] presented $\mathrm{C}$ means algorithm with 10 patients knee images.

Enumeration of JSW using an automatic method for 3 wrist joints is presented by Yinghe Huo in [19] to detect Rheumatoid arthritis with 50 radiographs of patients which is applied with consecutive searches of separate path segments. But the method doesn't work with wrist affected by sclerosis diseases. Hemavati $\mathrm{J}$ et al. in [18] compared four different methods related to the confusion matrix with, a true positive, true negative, false positive and false negative values with real-life patients data for diagnosis of arthritis with knee joints.

To predict RA using signal processing Lakshmi V et al. in [20] presented a system with input as patients genome arrangement of 30,000 nucleotide length. The RA variance index is $95 \%$ accurate for a variety of data genomic sequence.

To determine suitable drugs for Rheumatoid arthritis, Edgar .A et al. in [21] presented a system which uses neural network with real data from Saint's raphael hospital. The Recurrent neural network analysis the data based on LSTM and predicts the drugs for RA patients with an accuracy of $97 \%$. To diagnose Rheumatoid arthritis, Kento Morita in [22] proposed a method for finger joints carried out for 45 mild to severe patients. The method high variance estimation with erosion and JSN score of $50.9 \%$ and $64.3 \%$. But the method fails to detect the RA for 6 severe patients.

\section{RESEARCH METHODOLOGY}

The organized analysis is finished using Preferred Reporting Items for Systematic Reviews and Meta-Analysis (PRISMA) method which was introduced by Moher et al. [13]. PRISMA, a unique method that can benefit researchers doing organized reviews and meta-analysis accurately and also benefit to changes in an organized manner. In a organized evaluation with an precise, inclusive and organized investigation, diverse philosophies can be scrutinized and are issued in the procedural manner of outmoded articles by diverse researchers.

\subsection{Literature Search}

In the literature review, seven different databases were selected for systematic review: Hindawi, Springer, Taylor and Francis, IEEE, Elsevier, Wiley digital library and ACM. According to a well-defined objectives and exploratory questions in research, the document exploration was done on keyword as "machine learning" and "arthritis". Total of 148 articles from 2010 to 2018 is been retrieved for the review.

\subsection{Study Selection, Abstraction and Succinct of data}

Here in this stage, a result, based on search 148 articles were selected, since the suitable criteria essentially to include proper articles are, the research articles are curtained based on the presence (inclusion) and omission (exclusion) criteria. Information mining was designed to analyse, categorize and produce the suitable articles grounded on imperious defined criteria. At that moment based our investigation of information mining form, which could be achieved with the best outcomes with commendations. The criteria included the author details, publication year (from and to), journal or conference, type of disease, problem type and definition, objectives, research gaps, machine learning method, findings and results. The table demonstrates the inclusion and exclusion criteria.

Subsequently, revising and summarizing the collected full texts, 24 documents matched the eligible criteria that are suitable articles selected for analysis and interpretation. Assessors recited whole document body, acutely with including their particulars to select the documents associated to applying machine learning with arthritis. Nevertheless, moving to bestow the organized method and selecting the appropriate documents will take a huge amount of efforts with w.r.t time and cost, but because of the specific organization.

Here, we confirm utmost apposite and pertinent documents associated/ relevant to the topic/ domain of interest for this review.

Table 1: Criteria for document review

\begin{tabular}{|l|l|}
\hline \multicolumn{1}{|c|}{$\begin{array}{c}\text { Inclusion } \\
\text { Criteria }\end{array}$} & \multicolumn{1}{c|}{ Exclusion Criteria } \\
\hline $\begin{array}{l}\text { Language: } \\
\text { English }\end{array}$ & $\begin{array}{l}\text { Articles written in other languages } \\
\text { except English }\end{array}$ \\
\hline $\begin{array}{l}\text { Period: Year } \\
2010 \text { to } 2018\end{array}$ & Before 2010 \\
\hline
\end{tabular}




\begin{tabular}{|l|l|} 
& \\
\hline $\begin{array}{l}\text { Only original } \\
\text { articles from } \\
\text { conferences }\end{array}$ & $\begin{array}{l}\text { Articles related to treatment, clinical } \\
\text { decision and making process }\end{array}$ \\
\hline Human diseases & Diagnosis related to animals \\
\hline $\begin{array}{l}\text { Diagnosis using } \\
\text { machine learning }\end{array}$ & Books, book chapters and thesis \\
\hline
\end{tabular}

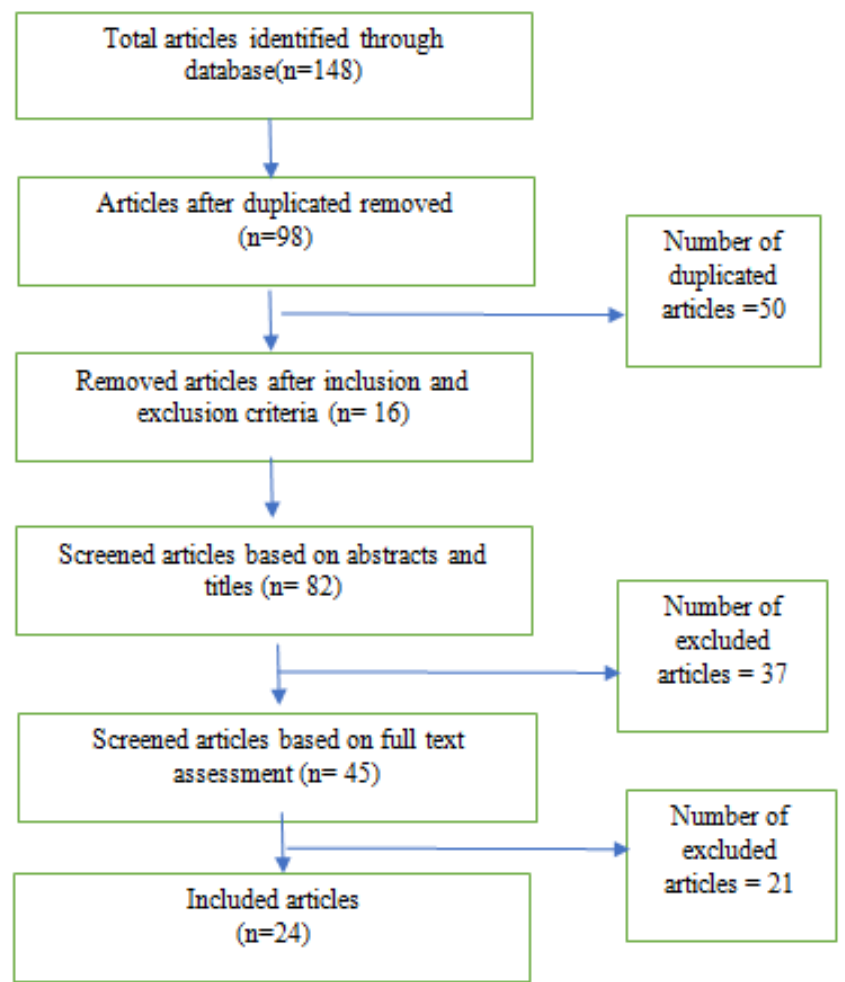

Fig:2 PRISMA method for review

\section{RESULTS}

The conclusions and analyzed articles are presented in this section. Through this review process, we can anticipate the efficiency of machine learning methods applied to Arthritis. The frequency of published articles from 2016 to 2018 is more in number. In this regard, almost $80 \%$ of papers from 2017 to 2018 are included. For previous few years, significant progress has stayed in machine learning for arthritis detection and diagnosis.

\subsection{Distribution of articles by publication year}

The included articles in analysis are retrieved from different publications from reputed international conventions between 2010 and 2018. The number of involved articles by their type of journal is obtainable in the figure. The figure specifies substantial upsurge in noted articles retrieved from 2015 till now. Within the year 2015, 12 articles were published, 17, 45 and 74 in the year 2016, 2017 and 2018 respectively.

Table 2: different data bases selected for research review

\begin{tabular}{|l|c|c|}
\hline $\begin{array}{c}\text { Database } \\
\text { Selected }\end{array}$ & $\begin{array}{c}\text { Number of } \\
\text { papers }\end{array}$ & Percentage \\
\hline Elsevier & 12 & 25.53 \\
\hline IEEE & 18 & 38.29 \\
\hline Springer & 4 & 8.51 \\
\hline
\end{tabular}

\begin{tabular}{|l|c|c|}
\begin{tabular}{|l|c|} 
Taylor and \\
Francis
\end{tabular} & 8 & 17.02 \\
\hline $\begin{array}{l}\text { Wiley online } \\
\text { library }\end{array}$ & 3 & 6.39 \\
\hline ACM & 1 & 2.13 \\
\hline Hindawi & 1 & 2.13 \\
\hline Total & $\mathbf{4 7}$ & $\mathbf{1 0 0}$ \\
\hline \multicolumn{2}{|c|}{17} \\
\hline
\end{tabular}

Fig 3: Distribution of articles

\subsection{Distribution of articles by the database provider}

In the review process, 7 databases are selected to search for articles. We can see the different information system providers in the table. IEEE has ranked 1st with $38.29 \%$, Elsevier with 2nd $25.53 \%$ and Taylor and Francis with $17.02 \%$.

\section{DISCUSSION}

The investigation was intended to review the machine learning influence on arthritis detection and diagnosis. The early research has noted the importance of machine learning, but only a few articles are published to scrutinize the extent of machine learning into arthritis diagnosis. As a result, this review can be seen as valuable to assess the impact of machine learning in arthritis diagnosis. The first question in this study was which method to be applied for an arthritis diagnosis. Therefore this review classified the application of machine learning with diverse perspective. Based on the analysis different methods are reviewed as follows: deep learning, random forest, decision trees, support vector machine and neural networks. The result shows the researchers have more tended towards deep learning method. We can't reach to conclusion by analyzing different types of diseases, so considered only one type of disease i.e. arthritis which can be diagnosed. One of the finding was that use of deep learning due to its ability to diagnose the disease. The investigation covers our systematic study through detailed knowledge around the effectiveness of machine learning in arthritis diagnosis. Therefore, current learning provides extra proof of study for investigators who work in healthcare domains. Additionally, using the outcomes of this study, it can be discovered that which methods to be included and which have been neglected. Another finding in the study is Artificial Intelligence (Convolution Neural Networks) method for disease diagnosis using genetic disorders or through radiology images to predict the future of patients. 


\section{INTERPRETATION}

The analysis has contended around preceding studies that are steered around machine learning methods in arthritis diagnosis. The study/ investigation, in the core area persisted to evaluate the consequences of machine learning methods and occurrences on refining diagnosis, to decline faults in diagnosis. So, performed the quest on the foremost aim. Due to this respect, seven reputed databases were selected which includes IEEE, Elsevier, ACM, Taylor and Francis, Springer, Wiley digital library and Hindawi to collect the reputed published articles in the period of 2010 to 2018. To reach the main goals of the study, all documents are collected, classified by publication from and to year, type and scopus indexed journals or type of conference and findings and gaps in research. The review shows that the rate of publications has been increased. The most noticeable finding is the use of machine learning in arthritis diagnosis. Yet another key point to recall is the classification of different machine learning methods represented as our results. Hence, the review is foundation for further research, arranged with summary of review papers with different methods are provided in Appendix A in order for future studies for researchers. $90 \%$ and more number of researchers provided a positive impact of applying machine learning to enhance and conduct diagnosis. Therefore in further studies, we consider some hybrid methods for an arthritis diagnosis. This learning has positively conducted with a systematic generalization of different machine learning methods, but with some limitations. Further, the study can be continued with still valued resources, such as book chapters and thesis.

\section{BIBLIOGRAPHY}

1. N. Armstrong, P. Hilton, Doing diagnosis: whether and how clinicians use a diagnostic tool of uncertain clinical utility, Soc. Sci. Med. 120 (2014) 208-214

2. J.L. Scully, What is a disease? EMBO Rep. 5 (2004) 650-653.

3. R. Leaman, R. Islamaj Do gan , Z. Lu , DNorm: disease name normalization with pairwise learning to rank, Bioinformatics 29 (2013) 2909-2917

4. R.H. Scheuermann, W. Ceusters, B. Smith, Toward an ontological treatment of disease and diagnosis, Summit Transl. Bioinform. 2009 (2009) 116-120.

5. P. Croft , D.G. Altman , J.J. Deeks , K.M. Dunn , A.D. Hay , H. Hemingway , L. LeResche , G. Peat , P. Perel , S.E. Petersen , R.D. Riley, I. Roberts , M. Sharpe , R.J. Stevens , D.A. Van Der Windt, M. Von Korff, A. Timmis, The science of clin- ical practice: disease diagnosis or patient prognosis? Evidence about "what is likely to happen"should shape clinical practice, BMC Med. 13 (2015) 20 .

6. Committee on Diagnostic Error in Health Care, Board on Health Care Services, Institute of Medicine, T.N.A.o, Sciences, Improving Diagnosis in Health Care, The National Academies Press, Washington, DC, 2015.

7. https://www.cdc.gov/arthritis/data_statistics/national-statistics.html

8. Fangjie Zhang, Role of osteopontin in rheumatoid arthritis ,Rheumatology International, April 2015, Volume 35, Issue 4, pp 589-595

9. B. Malmir , M. Amini , S.I. Chang, A medical decision support system for dis- ease diagnosis under uncertainty, Expert Syst. Appl. 88 (2017) 95-108

10. M. Nilashi, O. bin Ibrahim , H. Ahmadi, L. Shahmoradi , An analytical method for diseases prediction using machine learning techniques, Comput. Chem. Eng. 106, 106 (2017) 212-223 .

11. M. Nilashi, O. Ibrahim , H. Ahmadi, L. Shahmoradi , M. Farahmand , A hybrid intelligent system for the prediction of Parkinson's disease progression using machine learning techniques, Biocybern. Biomed. Eng. 38 (2018) 1-15.
12. M. Nilashi, H. Ahmadi , L. Shahmoradi , A. Mardani, O. Ibrahim , E. Yade- garidehkordi, Knowledge discovery and diseases prediction: a comparative study of machine learning techniques, J. Soft Comput. Decis. Support Syst. 4 (2017) 8-16.

13. D. Moher, L. Shamseer, M. Clarke, D. Ghersi , A. Liberati , M. Petticrew, P. Shekelle, L.A. Stewart, Preferred reporting items for systematic review and meta-analysis protocols (PRISMA-P) 2015 statement, Syst. Rev. 4 (2015).

14. Dr. Neeraj et al. Prediction of Arthritis using Classification and Regression Tree Algorithm, 2nd International Conference on Communication and Electronics Systems, ISBN:978-1-5090-5013-0, 2017

15. Min Chen et al. Disease Prediction by Machine Learning over Big Data from Healthcare Communities, IEEE. Translations and conten mining, DOI 10.1109/ACCESS.2017.2694446, 2016

16. $\mathrm{G}$ Dong et al. , Identification of candidate genes for Rituximab response in Rheumatoid Arthritis with Weighted Gene Co-expression Network Analysis, DOI: 978-1-5386-3524-7/17, 2017

17. Gobikrishnan M et al. , Diagnosis of Rheumatoid Arthritis in Knee using Fuzzy C Means Segmentation Technique, International Conference on Communication and Signal Processing, DOI: 9785090-0396-9/16, 2016

18. Hemavathi $\mathbf{J}$ et al. Knee Vibratography: Arthritis Diagnosis through Non-Invasive Cloud based Artificial Intelligence, 2nd IEEE International Conference on Computational Systems and Information Technology for Sustainable Solutions 2017, DOI: 978-1-5386-2044$1 / 17,2017$

19. Yinghe Huo et al. , Automatic quantification of radiographic wrist joint space width of patients with rheumatoid arthritis, DOI 10.1109/TBME.2017.2659223, 2016

20. Lakshmi T V et al., Design of a state-machine based genomic simulator and development of a system for prediction of Rheumatoid Arthritis (RA) using signal processing techniques, Proceedings of Second International conference on Circuits, Controls and Communications, DOI: 978-1-5386-0615-5/17, 2017

21. Parijata Majumdar et al., Detection of Inflammation from temperature profile using Arthritis knee joint Datasets, 2018 IEEE International Conference on Healthcare Informatics, DOI 10.1109/ICHI.2018.00077, 2017

22. Edgar A et al. , Medication adherence improvement on Rheumatoid Arthritis patients based on past medical records, DOI: 978-1-53867008-8/18, 2018

23. KENTO MORITA et al., COMPUTER-AIDED DIAGNOSIS SYSTEM FOR RHEUMATOID ARTHRITIS USING MACHINE LEARNING, Proceedings of the 2017 International Conference on Machine Learning and Cybernetics, DOI: 978-5386-0408-3/17, 2017

\section{AUTHORS PROFILE}

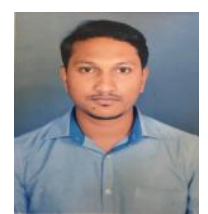

Sujeet More is Pursuing PhD from Lovely Professional University, Punjab.

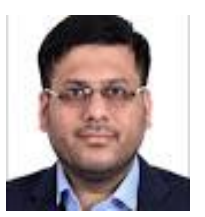

Jimmy Singla is working as Associate Professor in School of CSE, Lovely Professional University, Punjab 